\title{
Flexibilidad, equilibrio dinámico y estabilidad del core para la prevención de lesiones en deportistas universitarios
}

\author{
Flexibility, dynamic balance and core stability for injury prevention in university athletes
}

\author{
Andrés Villaquiran-Hurtado ${ }^{*}$ orcid.org/0000-0002-6156-6425 \\ Nancy Jannet Molano-Tobar ${ }^{2}$ orcid.org/0000-0003-1953-4101 \\ Enmanuel Portilla-Dorado² orcid.org/0000-0003-4331-7633 \\ Andrés Tello 3 orcid.org/0000-0001-6226-4229
}

1 Programa Fisioterapia, Universidad del Cauca. Popayán, Colombia

2 Programa Licenciatura en Educación Física, Recreación y Deportes, Universidad del Cauca. Popayán, Colombia

3 Comité Olímpico Chino. Beijing, China

\section{Resumen}

Introducción: El deporte competitivo demanda un intensivo acondicionamiento físico que conlleva a un mayor riesgo de lesión que afectan la salud, el rendimiento físico, la participación en los entrenamientos, las competencias y los logros deportivos. Objetivo: Determinar las características de la estabilidad del core, el equilibrio dinámico de miembros inferiores y la flexibilidad en deportistas universitarios. Materiales y métodos: Estudio de corte transversal, con 86 deportistas universitarios que corresponden al $25 \%$ de la población universitaria. Se evaluó la estabilidad del core, el equilibrio dinámico de miembros inferiores y flexibilidad de la cadena posterior, hombro y cadera; se tuvo en cuenta la guía de valoración para la prevención de lesiones del lineamiento de política pública en ciencias del deporte. Resultados: El 83,7\% de atletas presentaron un índice de masa corporal normal, el 47,5\% mostró una flexibilidad promedio en el Sit and Reach, sin presencia de retracciones en cadera. Respecto al core el $77,9 \%$ de los participantes presentaron una mala estabilidad y el $47 \%$ riesgo de lesión, producto de las diferencias entre las extremidades inferiores en el equilibrio dinámico. Conclusiones: Los resultados sugieren implementar programas de prevención de lesiones para deportistas universitarios.

Palabras clave: Equilibrio postural; rango de movimiento; deportistas; abdomen; rendimiento deportivo. (Fuente: DeCS, Bireme).

\begin{abstract}
Introduction: Competitive sports demand intensive physical conditioning that leads to an increased risk of injury, consequently affecting the health, physical performance, participation in training, competition, and sport achievements of athletes. Objective: To determine core stability, lower limb dynamic balance and flexibility characteristics in university athletes. Materials and methods: A cross-sectional study with 86 university athletes, which corresponds to $25 \%$ of the university population. Core stability, lower limb dynamic balance, and flexibility of the posterior chain, shoulders and hip region were evaluated. The assessment guide from the public policy guidelines in sports sciences for the prevention of injuries was taken into account. Results: $83.7 \%$ of athletes displayed a normal body mass index and $47.5 \%$ showed an average flexibility in the Sit and Reach test without the presence of hip retractions. Regarding the core, $77.9 \%$ of the participants showed a poor stability and $47 \%$ had a risk of injury due to the differences between the lower extremities in the dynamic balance. Conclusions: These results suggest implementing injury prevention programs for university athletes.
\end{abstract}

Key words: Postural balance; range of motion; athletes; abdomen; sports performance. (Source: DeCS, Bireme). 


\section{Introducción}

El deporte ha sido recomendado por los diferentes beneficios que presenta para el cuidado de la salud y la prevención de enfermedades no transmisibles, sin embargo, el deporte competitivo requiere de una excelente forma física, lo que conlleva a un mayor riesgo de lesión(1). Cuando estas lesiones aparecen, afectan considerablemente la salud, el rendimiento físico, la participación en los entrenamientos, las competencias y por ende, los logros deportivos ${ }^{(2)}$.

Estudios han demostrado la importancia de la prevención de lesiones, producto de la alta incidencia en que se presentan, por ejemplo, se estima que 7 millones de americanos anualmente reciben atención medica por lesiones deportivas, siendo entre los 15 y 24 años las edades con mayor afectación(3); mientras tanto en el departamento del Cauca (Colombia), un estudio reportó tasas de lesión de 0,6 por 1000 horas de entrenamiento, con una alta presencia de lesiones por sobreuso(4). Las lesiones pueden afectar las diferentes estructuras del sistema musculo esquelético, apareciendo cuando la carga aplicada a un tejido supera la tolerancia de resistencia, clasificándose tanto en agudas como por sobreuso(5). Las lesiones deportivas aparecen debido a diferentes mecanismos y factores, como los factores de riesgo intrínsecos, que incluyen el rango de movimiento articular(6), el control postural dinámico, la fuerza y la resistencia muscular del core( ${ }^{(7)}$.

Las distintas maniobras y acciones motrices en el deporte requieren de un alto control neuromuscular, por ejemplo una adecuada estabilidad dinámica de rodilla reduce el riesgo de lesión por no contacto(5,8,9); así como también el entrenamiento de la fuerza del core y su relación con la firmeza lumbopélvica, necesaria en la ejecución de cualquier gesto deportivo(10). Por ejemplo, en el fútbol el core es considerado un factor de riesgo intrínseco para la incidencia de lesión de rodilla, debido a las características y situaciones comunes de este deporte, como los cambios de dirección, las maniobras de deceleración y los aterrizajes en los saltos; las cuales generan un requerimiento importante de la estabilidad del tronco, la fuerza y la propiocepción del core ${ }^{(11)}$. En otros deportes como el tenis, la implementación de programas de entrenamiento del core ha sido asociada positivamente con el mejoramiento del equilibrio dinámico y la agilidad, generando un mejor rendimiento deportivo(12). Un estudio realizado a deportistas de taekwondo demostró que un entrenamiento del core de 8 semanas mejora la estabilidad dinámica producto de una mejor distribución de las presiones plantares, mejorando la condición física de los atletas(13). El core permite un mejor control neuromuscular en la generación y transferencia de la fuerza en el movimiento funcional de las extremidades inferiores, como en la anticipación sobre las fuerzas reactivas producidas en los diferentes movimientos deportivos. Por lo tanto, cualquier deficiencia en la estabilidad del core puede generar rápidamente la aparición de lesiones en los miembros inferiores(14).

En cuanto a la flexibilidad, existen evidencias que sugieren que el entrenamiento de la movilidad articular a diario podría ser beneficioso para la prevención de lesiones(15). Por ejemplo, la flexibilidad de los músculos isquiosurales en el fútbol influye notoriamente en el rendimiento sobre tareas, tales como; el sprint, la agilidad, el salto y el pateo(16). También, se ha establecido que una mejor flexibilidad con un adecuado uso de las diferentes técnicas de entrenamiento como el rodillo de espuma o la facilitación neuromuscular propioceptiva, pueden mejorar la eficiencia de la contracción muscular y la capacidad de salto en deportistas de fútbol sala(17). Dentro de estos elementos intrínsecos tanto la propiocepción, como la fuerza del core y la flexibilidad han sido herramientas utilizadas para la evaluación y desarrollo de los procesos de prevención(18).

Con relación a los modelos de prevención de lesiones, Van Machelen establece una secuencia para la implementación de estos programas; establecer la incidencia y severidad, establecer la etiología y los posibles mecanismos, introducir las medidas preventivas y valorar la efectividad son los pasos para la planeación, desarrollo y ejecución de estos procesos ${ }^{(19)}$. En consecuencia, la evaluación de estos componentes es fundamental para la planeación y desarrollo de los programas de prevención de lesiones, los cuales son cada vez más implementados por los profesionales de la salud y el deporte(1). Son pocos los estudios encontrados que realicen la valoración en conjunto de estos tres aspectos importantes de la prevención de lesiones en deportistas universitarios, por ello el objetivo del presente estudio fue determinar las características de la fuerza del core, la estabilidad dinámica de 
miembros inferiores y la flexibilidad en deportistas universitarios evaluados en la etapa de preparación física general.

\section{Materiales y métodos}

Estudio descriptivo, de corte transversal realizado a deportistas de una universidad pública del departamento del Cauca durante el primer periodo de 2018.

\section{Muestra}

En el estudio participaron 86 deportistas pertenecientes a los seleccionados universitarios entre los 17 y 33 años $(22,37$ años $\pm 3,77)$, de los cuales el $53,5 \%$ eran hombres, la distribución por deporte fue: 17 (19,8\%) de fútbol, 16 (18,6\%) fútbol sala masculino, $11(12,8)$ futbol sala femenino, 14 $(16,3 \%)$ de taekwondo, $16(18,6 \%)$ de tenis de campo, $6(7 \%)$ de voleibol y $6(7 \%)$ de patinaje. Los participantes debían cumplir con los siguientes criterios de inclusión: encontrarse activos en el sistema de registro académico universitario, hacer parte de los seleccionados en los diferentes deportes que realizan sus procesos de preparación deportiva y participación voluntaria. Como criterios de exclusión, se tuvo en cuenta que el deportista no completara la evaluación, retiro voluntario y lesión deportiva que impidiese la valoración, o lesión previa 6 meses antes de la valoración.

Para realizar la evaluación, se inició explicándole al deportista y entrenador los objetivos del estudio, se resolvieron preguntas y dudas que se pudiesen presentar, posteriormente, se procedió a la firma del consentimiento informado; luego el atleta diligenció el formato de evaluación en prevención de lesiones, elaborado por el grupo investigador y previamente ajustado mediante prueba piloto. Se recolectaron datos sociodemográficos, información deportiva, antecedentes de lesión y se procedió a la toma de medidas antropométricas, teniendo como referencia las recomendaciones establecidas por la Sociedad Internacional de Cineantropometría (ISAK) ${ }^{(20)}$. Para el cálculo del índice de masa corporal se utilizó la fórmula talla/peso corporal y para la clasificación se tomó como referencia los valores establecidos por la Organización Mundial de la Salud (OMS)(21). Para el análisis se utilizó la guía de evaluación descrita en los lineamientos de fisioterapia de la política pública en ciencias del deporte(17).

\section{Valoración de la flexibilidad}

Pruebas de Sit and Reach modificado: se realizó con el sujeto sentado con la espalda apoyada sobre una pared, con las caderas flexionadas y rodillas extendidas, con los brazos estirados y manos una encima de la otra. Una vez asumida esta posición se le pidió al deportista inclinarse hacia delante tratando de alcanzar la máxima distancia sin doblar las rodillas y con los pies juntos, manteniendo la posición durante 3 segundos, midiéndose la distancia alcanzada entre las manos y la punta de los pies(19).

Test de movilidad activa de hombro: Se usó para medir la integridad y el rango de movimiento escapular, combinando la rotación interna con aducción, la extensión con rotación externa, abducción y flexión, para la calificación de esta prueba se tuvo como referencia los parámetros dados por los Lineamientos de Política Pública en Ciencias del Deporte en Fisioterapia, dados por Coldeportes Nacional(22).

Test de Ely: El sujeto se colocó en decúbito prono, flexionando la rodilla hacia el glúteo, evaluando las dos extremidades inferiores ${ }^{(22) .}$

Test de Thomas: Se tuvo en cuenta si el sujeto extiende la rodilla y eleva ésta por encima de la cadera al hacer la maniobra, esto se considera positivo para acortamiento del musculo psoas(22).

Test de Ober: Permitió valorar si existe un acortamiento de la banda iliotibial $(22,23)$.

\section{Valoración del equilibrio dinámico}

Se utilizó el Star Exercusion Balance Test (SEBT), que consiste en alcanzar el punto más lejano que le sea posible, en cada una de las 8 direcciones, sobre una estrella dibujada en el piso; el alcance debe realizarse sin desequilibrios, ni compensaciones, repitiéndose 3 veces por cada dirección(24).

\section{Medición de la estabilidad del core}

Se realizaron 5 pruebas, cada una de las cuales tiene una puntuación 0 a 1 y la sumatoria de las cinco

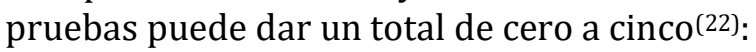

- Resistencia de flexores para evaluar el recto abdominal;

- Resistencia de extensores para examinar la musculatura extensora del tronco, el longuísimo y los multifidos; 
- $\quad$ Test de puente lateral por el lado derecho y puente lateral por el lado izquierdo, estas dos pruebas permiten la activación de los oblicuos externos, internos y el cuadrado lumbar; las pruebas consisten en soportar el peso corporal el máximo tiempo posible sosteniéndose sobre el antebrazo y el borde lateral del pie en de cubito lateral;

- Test de puente prono se hizo en decúbito prono soportando el peso del cuerpo sobre los antebrazos y los dedos de los pies, pidiéndosele al evaluado mantener la posición durante el mayor tiempo posible.

\section{Análisis estadístico}

Se aplicaron medios estadísticos como la prueba de Shapiro-Wilk para evidenciar la normalidad de la muestra, para el análisis descriptivo de los datos se obtuvieron medidas de tendencia central (media \pm desviación típica). Además, se llevó a cabo un análisis de comparación de medias entre distintas variables y un análisis correlacional por medio del test de Pearson (R), estableciendo el valor de significancia $\mathrm{p} \leq 0.05$. El tratamiento estadístico de los datos se llevó a cabo con el programa SPSS versión22 (Inc, Chicago, Illinois, Estados Unidos) para Windows.

\section{Consideraciones éticas}

Para la realización de la investigación se tuvo en cuenta los aspectos éticos, los cuales fueron validados por el Comité de Ética de la Vicerrectoría de Investigaciones de la Universidad del Cauca bajo el ID 4925, como la asociación de la declaración de Helsinki por la Asociación Americana Mundial (A.A.M) y de la Resolución 8430 del Ministerio de Salud, que establece las normas científicas y administrativas para la investigación en salud y de investigación con seres humanos en Colombia.

\section{Resultados}

La modalidad deportiva que albergó mayor población fue el fútbol sala con $31,4 \%$ de deportistas, seguido del futbol con 19,8\% y el de menor proporción fue el patinaje con $7 \%$. Con relación al índice de masa corporal se estableció una tendencia hacia la normalidad con un $83,7 \%$, y sólo 11 atletas se encontraron en sobrepeso. En la tabla 1, se registra la distribución de los deportistas con relación al deporte y el índice de masa Corporal.

La fase de entrenamiento en la que se encontraban al momento de realizar las evaluaciones fue la precompetitiva $33,7 \%$ y la fase competitiva $25,6 \%$. Con relación a los antecedentes de lesión, las patologías de mayor incidencia fueron en ligamento $12,8 \%$ y músculo $8,1 \%$, donde los miembros inferiores fueron la zona con mayor afectación $(26,7 \%)$ seguido de lesiones en el tronco $(3,5 \%)$. Se estableció que el deporte que más presentó estructuras lesionadas fue el futbol sala con tendencia a lesiones en la parte ligamentaria.

En referencia a los diferentes test aplicados para evaluar la flexibilidad musculo-esquelética se encontró en primer lugar, calificaciones promedio $(47,5 \%)$, seguidos de valores buenos $(38,4 \%)$, en la Tabla 2 se muestra como respondieron al test los deportistas.

Respecto al test de Ely, ambas extremidades presentaron una calificación negativa de $87,2 \%$ para el miembro inferior derecho, mientras que para la extremidad inferior izquierda el porcentaje encontrado fue de $88,4 \%$, situación similar para la prueba de Thomas donde se presentaron resultados negativos tanto en la extremidad izquierda $(70,9 \%)$ como para la derecha $(65,1 \%)$. En el test de Ober también se halló un predominio de la calificación negativa para ambas extremidades con 89,5\%; para el test de hombro se determinó que la mayor calificación encontrada fue de 3 en ambos hombros (derecho $57 \%$ e izquierdo $44,2 \%$ ), mientras que el $33,7 \%$ y el $34,9 \%$ presentaron calificación de 2 tanto para el hombro derecho y hombro izquierdo, respectivamente.

Al analizar las evaluaciones correspondientes a la estabilidad del core en todas las posiciones se evidenció que la calificación "malo" es la predominante en las pruebas $(77,9 \%)$ (Tabla 3 ). Para el equilibrio dinámico el $47,7 \%$ de miembros inferiores presentan niveles de riesgo para lesión, en la Tabla 4 se aprecia la distribución de esta variable con relación a los diferentes deportes evaluados.

Las correlaciones encontradas bajo el estadístico de Pearson ( $p \leq 0,05)$, se presentan en la Tabla 5, evidenciando las de muy alta y alta correlación, cabe notar que el test de Ely presentó alta correlación con el test de Thomas tanto de lado derecho como de lado izquierdo, donde las variables demuestran una significancia estadística. No se presentan las otras variables, ya que los hallazgos no manifiestan relevancia estadística. 
Tabla 1. Distribución de la población según el índice de masa corporal

\begin{tabular}{lcccc}
\hline \multicolumn{1}{c}{ Clasificación IMC } & Bajo Peso o Desnutrición (\%) & Normal (\%) & Sobrepeso (\%) & Total (\%) \\
\hline Taekwondo & 1,2 & 11,6 & 3,5 & 16,3 \\
Tenis de Campo & 1,2 & 15,1 & 2,3 & 18,6 \\
Voleibol & 0 & 5,8 & 1,2 & 7 \\
Fútbol Sala & 0 & 27,9 & 3,5 & 31,4 \\
Fútbol & 0 & 18,6 & 1,2 & 19,8 \\
Patinaje & 1,2 & 4,7 & 1,2 & 7 \\
Total & 3,5 & 83,7 & 12,8 & 100 \\
\hline
\end{tabular}

Tabla 2. Calificación de la prueba Sit and Reach en deportistas universitarios

\begin{tabular}{lcccccc}
\hline $\begin{array}{l}\text { Calificación Prueba Sit and } \\
\text { Reach }\end{array}$ & $\begin{array}{c}\text { Deficiente } \\
(\%)\end{array}$ & Pobre (\%) & $\begin{array}{c}\text { Promedio } \\
(\%)\end{array}$ & Bueno (\%) & $\begin{array}{c}\text { Excelente } \\
\text { (\%) }\end{array}$ & Total (\%) \\
\hline Taekwondo & 1,2 & 0 & 2,3 & 9,3 & 3,5 & 16,3 \\
Tenis de Campo & 0 & 3,5 & 11,6 & 3,5 & 0 & 18,6 \\
Voleibol & 0 & 0 & 1,2 & 5,8 & 0 & 7 \\
Fútbol Sala & 3,5 & 1,2 & 13,9 & 12,8 & 0 & 31,4 \\
Fútbol & 0 & 0 & 12,7 & 7 & 0 & 19,7 \\
Patinaje & 1,2 & 0 & 5,8 & 0 & 0 & 7 \\
Total & 5,9 & 4,7 & 47,5 & 38,4 & 3,5 & 100 \\
\hline
\end{tabular}

Tabla 3. Calificación de la estabilidad del core en deportistas universitarios

\begin{tabular}{lcccccc}
\hline & Regular & $\begin{array}{c}\text { Calificación del core } \\
\text { Malo (\%) }\end{array}$ & $\begin{array}{c}\text { Muy bueno (\%) } \\
(\%)\end{array}$ & $\begin{array}{c}\text { Excelente } \\
\text { (\%) }\end{array}$ & Total (\%) \\
\hline Taekwondo & 50 & 28,6 & 7,1 & 7,1 & 7,1 \\
Tenis campo & 68,8 & 31,3 & 0 & 0 & 0 & 0 \\
Voleibol & 50 & 16,7 & 33,3 & 0 & 7,4 \\
Fútbol sala & 85,2 & 7,4 & 0 & 0 & 0 \\
Fútbol & 100 & 0 & 0 & 0 & 0 & 3 \\
Patinaje & 100 & 0 & 0 & 0 & $3,5 \%$ & $100 \%$ \\
Total & 67 & 12 & 3 & 1 & & \\
\hline
\end{tabular}

Tabla 4. Riesgo de lesión de miembros inferiores- Test de SEBT

\begin{tabular}{llll}
\hline & Sin Riesgo (\%) & Riesgo (\%) & \multicolumn{1}{c}{ Total (\%) } \\
\hline Taekwondo & 10,5 & 5,8 & 16,3 \\
Tenis de Campo & 8,1 & 10,5 & 18,6 \\
Voleibol & 3,5 & 3,5 & 7 \\
Fútbol Sala & 17,4 & 14,0 & 31,4 \\
Fútbol & 10,5 & 9,3 & 19,8 \\
Patinaje & 2,3 & 4,7 & 7,0 \\
Total & 52,3 & 47,7 & 100 \\
\hline
\end{tabular}


Tabla 5. Correlaciones de las variables en deportistas universitarios

\begin{tabular}{lccc}
\hline \multicolumn{1}{c}{ Variables } & & Correlaciones & \\
\cline { 2 - 3 } & Significancia & Correlación & Interpretación \\
\hline $\begin{array}{l}\text { Test de Ely Derecho vs Test de Ely } \\
\text { Izquierdo }\end{array}$ & $<0,0001$ & 0,839 & Muy alta Correlación \\
$\begin{array}{l}\text { Test de Ely Derecho vs Test Thomas } \\
\text { Izquierdo }\end{array}$ & 0,006 & 0,692 & Alta \\
$\begin{array}{l}\text { Test de Ely Izquierdo vs Test Thomas } \\
\text { Derecho }\end{array}$ & $<0,0001$ & 0,419 & Moderada \\
$\begin{array}{l}\text { Test de Ely Izquierdo vs Test Thomas } \\
\text { Izquierdo }\end{array}$ & $<0,0001$ & 0,407 & Moderada \\
$\begin{array}{l}\text { Test de Movilidad Activa de Hombro } \\
\text { Derecho vs Test de Movilidad Activa de }\end{array}$ & $<0,0001$ & 0,695 & Alta \\
$\begin{array}{l}\text { Hombro Izquierdo } \\
\text { Test SEBT Derecho vs Test SBET }\end{array}$ & & & \\
$\begin{array}{l}\text { Izquierdo } \\
\text { Test SEBT Derecho vs Test de Thomas } \\
\text { Izquierdo }\end{array}$ & $<0,0001$ & 0,682 & Alta \\
\hline
\end{tabular}

\section{Discusión}

Los resultados del índice de masa corporal son adecuados en la mayoría de atletas universitarios, sobre esto cabe resaltar que una capacidad morfofuncional adecuada para la práctica del deporte se constituye en uno de los pilares básicos para rendir deportivamente, así como también una relación inadecuada entre el peso y la talla puede ser una medida cuantitativa que puede relacionar el entrenamiento, la nutrición y el desempeño del atleta(25). Al respecto, en una muestra de jugadoras de balón mano con un promedio de edad muy similar a la de este estudio, se encontró que todas las deportistas se encontraban con un IMC normal, resultados muy parecidos a los encontrados en este estudio donde tan solo 6 deportistas se encontraban por encima de los valores de normalidad(26).

Cabe resaltar que las características antropométricas dependen de cada modalidad deportiva, las particularidades de cada deporte, el nivel de competencia, el desarrollo madurativo y el ambiente donde se desarrollen las prácticas(27). Sin embargo, para determinar sobrepeso $\mathrm{u}$ obesidad en los deportistas se debe utilizar la toma de medidas como los pliegues cutáneos o la relación cintura/ cadera(28), esto podría complementar y mejorar próximos estudios que involucren la evaluación antropométrica en atletas universitarios.

Los deportistas presentaron mayores antecedentes de lesión en los miembros inferiores, con mayor afectación ligamentaria y muscular; en un estudio realizado en atletas caucanos se encontró resultados muy similares, reportando una incidencia mayor en los miembros inferiores, donde la lesión de ligamento fue una de las patologías reportadas con mayor frecuencia(4); frente a este tema el conocimiento de las diferentes lesiones sufridas por los atletas, la región anatómica y la zona corporal afectada pueden ser importantes en la planificación y desarrollo de los programas de prevención, aunque debe complementarse con la identificación de los factores de riesgo intrínseco y extrínseco que permitan tomar las mejores decisiones ${ }^{(9)}$.

En el test de Sit and Reach, la flexibilidad presentó una calificación promedio, aunque sin presencia de retracciones en la musculatura de cadera, situación que puede ser o no favorable en los deportistas. Al respecto Correa, et al.(29) encontraron una relación positiva entre los deportistas más flexibles y la probabilidad de lesión, demostrando que la flexibilidad no es un factor protector de lesión deportiva. Por su parte Sainz de Baranda et al.(30) contrario a lo anterior, afirman que conocer los niveles de flexibilidad permitirá optimizar el rendimiento y recuperar los arcos de movimiento por medio de programas preventivos específicos. El uso de diferentes programas de estiramiento en la práctica deportiva es habitual y tiene como objetivo mantener o mejorar el rango de movimiento de las articulaciones, disminuir el dolor, aumentar la tolerancia al estiramiento, ayudar con la recuperación del organismo, objetivos que permiten 
disminuir el riesgo de lesiones y mejorar el rendimiento del atleta(26). En relación con la flexibilidad de hombro, los universitarios presentaron un nivel de movilidad articular óptimo, pudiendo esto ser un factor protector de la articulación, por el contrario, un pectoral retraído, una restricción de la movilidad escapular y una reducción de la flexión de hombro puede ser la causa principal de las diferentes disfunciones de hombro(31,32).

Frente a los datos encontrados en la estabilidad del core, los deportistas universitarios mostraron resultados deficientes, situación que ha sido relacionada con el déficit del control neuromuscular, aparición de patologías de columna y miembros inferiores(15). Refiriéndose a lo anterior, Rivera afirma que los músculos del core juegan un papel muy importante en el comportamiento de las extremidades inferiores, puesto que cualquier deficiencia puede incrementar la fatiga, reducir el rendimiento y favorecer la aparición de lesiones en corredores(33).

Las mediciones de control postural dinámico, fuerza y resistencia del core también pueden ser herramientas utilizadas para la identificación de factores El déficit de la musculatura del core demostrado mediante pruebas clínicas han evidenciado un alto nivel de predicción de lesiones de las extremidades superiores, aunque deben considerarse para próximos estudios, la inclusión de la valoración de otros factores de riesgo como la movilidad articular, la fuerza muscular del miembro superior y la evaluación del entorno(34). Por su parte, Schroeder et al., afirman que el trabajo de la fuerza del core favorece el control postural, las habilidades de acción y reacción, y la estabilidad dinámica en las diferentes acciones deportivas, especialmente en deportes de contacto como el Judo(35). De acuerdo a lo anterior y con los resultados encontrados en los deportistas universitarios participantes de este estudio, se hace urgente implementar las medidas preventivas necesarias que ayuden a mejorar la estabilidad, fuerza y resistencia de la musculatura del core.

Con relación a la estabilidad dinámica de miembros inferiores, un déficit en el control postural dinámico entre las extremidades inferiores puede contribuir a un mayor riesgo de lesión de rodilla y tobillo(14). Sobre esto, Hartley et al., encontraron que un pobre rendimiento en el alcance anterior en el test de balance dinámico y un IMC inadecuado pueden asociarse con lesión de tobillo(36). Plisky et al.(37) encontraron un riesgo 6,5 veces mayor de sufrir lesión en basquetbolistas cuando las diferencias en los alcances eran 2,5 veces mayores entre las extremidades. Respecto al test de SEBT, el alcance en dirección anterior puede ayudar a identificar lesiones sin contacto en la rodilla o tobillo en atletas universitarios(38).

Por otra parte, la correlación existente entre las variables de flexibilidad manifestaron una alta significancia, lo cual determina que su aplicación es importante en el ámbito deportivo y un indicador para determinar la eficiencia en la medición, hecho que es corroborado en estudios de Ortega et al.(39), donde señalan que los estiramientos incrementan el rango de movilidad articular (ROM), lo cual fomenta los cambios en la longitud y rigidez de la unidad músculo-tendinosa, de la misma manera el hecho de evidenciar correlación entre los mismos test, permite establecer su validez como lo indica Cejudo et al.(40) al informar que un test es eficiente desde su aplicación y comprobación estadística, por lo tanto es importante promover en otros estudios comparaciones de protocolos, para potencializar los efectos que desde la aplicación de estiramientos musculares beneficia la salud.

\section{Conclusiones}

Los deportistas universitarios presentaron déficit en la estabilidad del core, el equilibrio dinámico y la flexibilidad de la cadena posterior, aunque se encontraron resultados favorables para la flexibilidad de la musculatura de hombro, la banda iliotibial y los flexores de cadera. Lo anterior permite identificar los posibles factores de riesgo que presentan los atletas para poder tomar decisiones necesarias y oportunas en la planeación, organización y ejecución de planes de prevención de lesiones deportivas específicos para atletas universitarios.

\section{Recomendaciones}

Para próximos estudios es necesario ampliar la muestra de estudio y evaluar otros factores relacionados con la lesión, también se sugiere incluir otras variables antropométricas que permitan una investigación más completa en este aspecto. Sin embargo, estos resultados pueden ser de gran aporte para entrenadores, fisioterapeutas y profesionales del deporte siendo de gran utilidad para evaluar las 
diferentes capacidades que influyen en el rendimiento y la salud del deportista universitario.

Conflicto de intereses: Los autores declaran no tener ningún conflicto de intereses.

\section{Referencias}

1. Bakken A, Targett S, Bere T, Adamuz M, Tol J, Whiteley R, et al. Health conditions detected in a comprehensive periodic health evaluation of 558 professional football players. Br J Sport Med. 2016; 50:1142-50. Disponible en: http://dx.doi.org/10.1136/bjsports-2015-095829

2. Vriend I, Gouttebarge V, Finch C, Van Mechelen W, Verhagen E. Intervention Strategies Used in Sport Injury Prevention Studies: A Systematic Review Identifying Studies Applying the Haddon Matrix. Sport Med. 2017; 47(10):2027-43. Disponible en: http://dx.doi.org/10.1007/s40279-0170718-y

3. Conn J, Annest J, Gilchrist J. Sports and recreation related injury episodes in the US population. Injury Prevention. 2003; 9:117-23. http://dx.doi.org/10.1136/ip.9.2.117

4. Villaquiran A, Portilla-Dorado E, Vernaza-Pinzón P. Caracterización de la lesión deportiva en atletas caucanos con proyección a Juegos Deportivos Nacionales. Revista Universidad y Salud. 2016; 18(3):541-9. Disponible en: http://dx.doi.org/10.22267/rus.161803.59

5. Dennis R, Finch C. Sports Injuries. International Encyclopedia of public health (Second Edition). 2017: 7986. Disponible en: https://dx.doi.org/10.1016/B978-0-12803678-5.00432-X.

6. Storm JM, Wolman R, Bakker E, Wyon M. The relationship between range of motion and injuries in adolescent dancers and sportspersons: A systematic review. Front Psychol. 2018; 9:287. Disponible en: https://dx.doi.org/10.3389/fpsyg.2018.00287

7. De Blaiser C, Ridder R, Willems T, Vanden L, Danneels L, Roosen P. Impared core stability as risk factor for the development of lower extremity overuse injuries: A prospective cohort study. Am J Sports Med. 2019; 47(7):1713-21. Disponible

en: https://dx.doi.org/10.1177/0363546519837724

8. Markstrom J, Grip H, Schelin L, Hager C. Dynamic knee control and movement strategies in athletes and nonathletes in side hops: Implications for knee injury. Scand J Med Sci Sports. 2019. Disponible en: https://dx.doi.org/10.1111/sms.13432

9. Van Mechelen W, Hlobil H, Kemper H. Incidence, Severity, Aetiology and Prevention of Sports Injuries: A Review of Concepts. Sport Med. 1992; 14(2):82-99. https://www.ncbi.nlm.nih.gov/pubmed/1509229

10. Coulo M, Silva M, Manfredini B. Strategies for injury prevention in Brazilian football: Perceptions of physiotherapists and practices of premier league teams. Phys Ther Sport. 2017. Disponible en: http://dx.doi.org/10.1016/j.ptsp.2017.07.004

11. Alentorn-Geli E, Myer G, Silvers H, Samitier G, Romero D, Lázaro-Haro $\mathrm{C}$, et al. Prevention of non- contact anterior cricuate ligament injuries in soccer players. Part 1: Mechanisms of injury and underlying risk factors. Knee Surg Traumatol Arthrosc. 2009; 17(7):705-29. Disponible en: http://dx.doi.org/10.1007/s00167-009-0813-1
12. Bashir S, Nuhmani S, Dhall R, Muaidi Q. Effect of core training on dynamic balance and agility among indian junior tennis players. J Back Musculoskelet Rehabil. 2019; 32(2):245-252. Disponible http://dx.doi.org/10.3233/BMR-170853

13. Yoon S, Sung D, Park G. The effect of active core exercise on fitness and foot pressure in Taekwondo club students. J Phys Ther Sci. 2015; 27(2):509-11. Disponible en: http://dx.doi.org/10.1589/jpts.27.509

14. Yeung J, Cleves A, Griffiths H, Nokes L. Mobility, proprioception, Strength and FMS as predictors of injury in professional footballers. BMJ Sport and Exercise Medicine. 2016; 2:1-6. Disponible en: http://dx.doi.org/10.1136/bmjsem-2016-000134

15. Vera-García F, Barbado D, Moreno-Perez V, HérnandezSánchez S, Juan-Recio C, Elvira J. Core stability. Concepto y aportaciones al entrenamiento y la prevención de lesiones. Rev Andal Med Deporte. 2015; 8(2):79-85. Disponible en: http://dx.doi.org/10.1016/j.ramd.2014.02.004

16. García- Pinillos F, Ruiz- Ariza A, Moreno del Castillo R, Latorre- Róman PÁ. Impact of limited hamstring flexibility on vertical jump, kicking speed, sprint, and agility in young football players. J Sports Sci. 2015; 33(12):1293-7. Disponible http://dx.doi.org/10.1080/02640414.2015.1022577

17. Portilla-Dorado E, Villaquiran-Hurtado A, Molano- Tobar N. Potencia del salto en jugadores de fútbol sala después de la utilización del rodillo de espuma y la facilitación neuromuscular propioceptiva en la musculatura isquiosural. Rev Acad Colomb Cienc EX Fis Nat. 2019; 43(167):165-76. Disponible en: http://dx.doi.org/10.18257/raccefyn.846

18. De Blaiser C, Roosen P, Willems T, Danneels L, Bossche L, De Ridder R. Is core stability a risk factor for lower extremity injuries in an athletic population? Asystematic review. Phys Ther Sport. 2018; 30:48-56. Disponible en: http://dx.doi.org/10.1016/j.ptsp.2017.08.076

19. Ayala F, Sainz de Baranda P, de Ste Croix M, Santoja F. Fiabilidad y validez de las pruebas sit-and-reach: revisión sistemática. Rev Andaluza Med Deporte. 2012; 5(2):57-66. Disponible en: https://www.elsevier.es/es-revista-revistaandaluza-medicina-del-deporte-284-articulo-fiabilidadvalidez-pruebas-sit-and-reach-revisionX1888754612495328

20. Stewart A, Marfell-Jones M, Olds T. Protocolo Internacional para la Valoración Antropométrica ISAK. 2011.

21. Organización Mundial de la Salud. Obesidad y Sobrepeso. [Fecha de consulta: 18 mayo 2019]. 2018. Disponible en: https://www.who.int/es/news-room/factsheets/detail/obesity-and-overweight

22. Departamento Administrativo del deporte la recreación y el aprevechamiento del tiempo libre. Lineamientos de politica pública en ciencias del deporte en Fisioterapia. 2015; 1-349.

23. Cook G, Burton L, Hoogenboom BJ. Functional Movement Screening: The Use of Fundamental Movements as an Assessment of function- part 2. The International Journal Sports Physical Therapy. 2014; 9(4):549-63. Disponible en: https://www.ncbi.nlm.nih.gov/pmc/articles/PMC4060319 /

24. Gribble PA, Hertel J. Considerations for Normalizing Measures of the Star Excursion Balance Test. Meas Phys Educ Exerc Sci. 2003; 7(2):89-100. Disponible en: https://doi.org/10.1207/S15327841MPEE0702_3

25. Rivera-Sosa J, Muñoz-Daw M, Cervantes- Borunda M, Romero-Martinez C N-LR. Análisis cineantropométrico de 
base en atletas universitarios mexicanos, participantes en la Universiada Nacional 2010. Rev Mex Investig en Cult Fis y Deport. 2012; 4(5):168-85.

26. Ramos-Angulo A, Medina-Porqueres I, Ortiz-Bish A, RuizMartinez Y, Medina-Jimenez L, Elena-Gamboa J. Perfil antropométrico de jugadoras de balonmano femenino de élite. Rev Andal Med Deporte. 2018; 11(2):47-51. Disponible https://dx.doi.org/10.1016/j.ramd.2016.09.002

27. Monteiro-Texeira D, Del Fraro J, Soares F, Reeberg L, Simoes C, Petroski E. Las características antropométricas de los deportistas de élite de los equipos brasileños juvenil y adulto de voleibol. Rev Andal Med Deporte. 2016; 9(4):16065. Disponible

en:

https://dx.doi.org/10.1016/j.ramd.2015.05.007

28. Canda A. Deportistas de alta competicón con índice de masa corporal igual o mayor a $30 \mathrm{~kg} / \mathrm{m} 2$. ¿Obesidad o gran desarrollo muscular?. Apunts Med Esport. 2017; 52(193):29-36. Disponible en: https://dx.doi.org/10.1016/j.apunts.2016.09.002

29. Correa-Mesa J, Rodriguez-Camacho D, Camargo-Rojas D Correa-Morales J. Prevalencia de lesiones en luchadores olímpicos pertenecientes a la Liga de Lucha Olímpica de Bogotá, D.C. Rev Fac Med. 2016; 64:99-104. Disponible en: http://dx.doi.org/10.15446/revfacmed.v64n3Supl.50971

30. Sainz de Baranda P, Cejudo A, Ayala F SF. Perfil de la flexibilidad de la extremidad inferior en jugadoras senior de fútbol sala. Rev Española Educ Física y Deport. 2015; 409(7):35-48. Disponible en https://www.reefd.es/index.php/reefd/article/view/86

31. Ayala F, Saniz de Baranda P, Cejudo A. El entrenamiento de la flexibilidad: técnicas de estiramiento. Rev Andal Med Deporte. 2012; 5(3):105-112. Disponible en: https://dx.doi.org/10.1016/S1888-7546(12)70016-3

32. Reeser JC, Joy EA, Porucznik CA, Berg RL, Colliver EB, Willick SE. Risk Factors for Volleyball-Related Shoulder Pain and Dysfunction. PMRJ. 2010; 2(1):27-36. Disponible en: http://dx.doi.org/10.1016/j.pmrj.2009.11.010

33. Rivera C. Core and lumbopelvic stabilization in runners. Phys Med Rehabil Clin N Am. 2016; 27(1):319-37. Disponible http://dx.doi.org/10.1016/j.pmr.2015.09.003

34. Silfies S. Ebaugh D, Pontillo M, Butowicz C. Critical review of the impact of core stability on upper extremity athletic injury and performance. Braz J Phys Ther. 2015; 19(5):3608. Disponible en: http://dx.doi.org/10.1590/bjptrbf.2014.0108

35. Schroeder H, Dero D, Oliveira J, Cidral J, Shiguemi A, Viseux F, Fernandes D, et al. Effects of core strengthening on balance in university judo athletes. J of Bodywork and Movement Therapies. 2019. Disponible en: https://dx.doi.org/10.1016/j.ptsp.2018.08.008

36. Hartley E, Hoch M, Boling M. Y-balance test performance and BMI are associated with ankle sprain injury in collegiate male athletes. J Sci Med Sport. 2018; 21(7):676-80. Disponible https://dx.doi.org/10.1016/j.jsams.2017.10.014

37. Plisky P, Rauh M, Kaminski T, Underwood F. Star Excursion Balance Test as a Predictor of Lower Extremity Injury in High School. Journal of Orthopedic and Sports Physical Therapy. 2006; 36(12):911-19. Disponible en: https://dx.doi.org/10.2519/jospt.2006.2244

38. Stiffler M, Bell DR, Sanfilippo J, Hetzel S, Pickett K, Heiderscheit B. Star excursion balance test anterior asymetry is associated with injury status in division I collegiate athletes. J Orthop Sports Phys Ther. 2017; 47(5):339-46. Disponible https://dx.doi.org/10.2519/jospt.2017.6974

39. Ortega C, Menendez H, Herrero AJ. Cambios a corto plazo del estiramiento estático, la electroestimulación y las vibraciones de cuerpo completo en la flexibilidad de los isquiotibiales. CCD. 2019; 15:43-50. Disponible en: http://dx.doi.org/10.12800/ccd.v14i40.1224

40. Cejudo Palomo A, San Cirilo B, Robles-Palazón F, Saiz De Baranda M. Análisis del perfil de flexibilidad en jóvenes taekwondistas. Rev Artes Marciales Asiáticas. 2018; 13(2s):30. Disponible en: http://revpubli.unileon.es/ojs/index.php/artesmarciales/a rticle/view/5503 